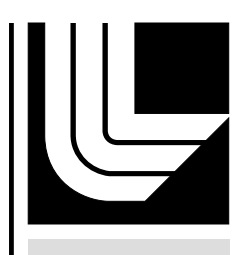

LAW RENCE LIVERMORE N A T IO N A L LABORATORY

\title{
The status of open heavy flavor production at $\mathrm{RHIC}$
}

R. Vogt

January 12,2011

Quarkonium 2010: Three Days of Quarkonium Production in $\mathrm{pp}$ and $\mathrm{pA}$ Collisions

Palaiseau, France

July 29, 2010 through July 31,2010 
This document was prepared as an account of work sponsored by an agency of the United States government. Neither the United States government nor Lawrence Livermore National Security, LLC, nor any of their employees makes any warranty, expressed or implied, or assumes any legal liability or responsibility for the accuracy, completeness, or usefulness of any information, apparatus, product, or process disclosed, or represents that its use would not infringe privately owned rights. Reference herein to any specific commercial product, process, or service by trade name, trademark, manufacturer, or otherwise does not necessarily constitute or imply its endorsement, recommendation, or favoring by the United States government or Lawrence Livermore National Security, LLC. The views and opinions of authors expressed herein do not necessarily state or reflect those of the United States government or Lawrence Livermore National Security, LLC, and shall not be used for advertising or product endorsement purposes. 


\title{
The status of open heavy flavor production at RHIC
}

\author{
R. Vogt ${ }^{\mathrm{a}, \mathrm{b}}$ \\ ${ }^{a}$ Physics Divsion, Lawrence Livermore National Laboratory, Livermore, CA 94551 \\ ${ }^{b}$ Physics Department, University of California at Davis, Davis, CA 95616
}

\begin{abstract}
We discuss the calculation of open heavy flavor cross sections at RHIC and describe how the semileptonic decays of charm and bottom quarks can be separated.

Keywords: heavy flavor, production
\end{abstract}

Heavy flavor production is an important complementary measurement to 'hidden' flavor (quarkonium) production. At the most basic level, the calculation of the quarkonium production cross section in the color evaporation model [1] is based on parameters set from evaluation of the open heavy flavor cross section. It was shown some time ago that the energy dependence of the open charm and charmonium production cross section was essentially the same, both for photoproduction and hadroproduction [2]. Thus a good understanding of the energy dependence of the charm cross section is important to fix the energy dependence of the $J / \psi$ cross section. We use the same mass, factorization scale, renormalization scale and set of parton densities to fix the scale factor between the open charm cross section below the $D \bar{D}$ threshold and the inclusive $J / \psi$ cross section.

Heavy flavor production is rather unique in that the total heavy flavor production cross section is analytically calculable to leading order at the partonic level. In addition the finite heavy flavor mass provides a scale to control the transverse momentum distributions down to $p_{T} \rightarrow 0$. In addition, because charm and bottom quarks are massive, they are produced almost exclusively in the initial parton-parton interactions in heavy-ion collisions. In the absence of any nuclear effects, the heavy flavor cross sections in $p A$ and $A A$ collisions would simply scale with the number of binary collisions. Thus departures from binary scaling for heavy flavor production provides information about nuclear effects. These can be divided into two categories: effects due to embedding the colliding partons in a nucleus (cold matter effects) and effects due to the large energy density in the

Email address: vogt@physics.ucdavis.edu (R. Vogt) final state in $A A$ collisions. The study of dense matter effects requires a detailed understanding of cold matter effects so that they can be unfolded from the dense matter effects. For more details, see the report from Working Group 2.

A number of dense matter effects on heavy flavor production have been predicted. Some of these do not change the total cross section but, instead, modify the $p_{T}$ spectra of heavy flavor hadrons and their decay products.

Heavy quark energy loss $[3,4,5,6,7]$ by collisional and radiative processes steepens the $p_{T}$ distribution relative to that in $p p$ collisions. On the other hand, random $p_{T}$ kicks result in transverse momentum broadening, increasing the average $p_{T}$ in both cold nuclear matter [8] and in passage through hadron bubbles in the mixed phase of a QGP [9]. If the medium surrounding the heavy quarks after production exhibits collective motion, such as transverse flow $[10,11]$, the low $p_{T}$ heavy quarks $\left(p_{T}<m\right)$ may be caught in this flow. Strong effects of energy loss $[12,13]$ on heavy flavor decays to electrons and charm flow [13] have already been seen in $\mathrm{Au}+\mathrm{Au}$ collisions at RHIC. Studying heavy flavor energy loss using single electrons requires being able to separate electrons from $c$ and $b$ decays since the large bottom and charm quark mass difference suggests that bottom quark energy loss is weaker than that for charm [3]. Similar energy-loss effects may be seen on $J / \psi$ production although the magnitude of the effects may differ because the $J / \psi$ is a heavy-heavy system rather than a heavy-light system.

Some QGP studies require accurate baseline determinations of the total heavy flavor cross sections to interpret other effects. For example, if more than one $c \bar{c}$ pair 
is produced in an $A A$ event, uncorrelated $c$ and $\bar{c}$ quarks might coalesce to form a $J / \psi$ in a QGP $[14,15,16,17]$. The total $c \bar{c}$ yield is needed to normalize the $J / \psi$ production rate from this process.

Unfortunately, the uncertainties on the open charm cross section, both measured and calculated, are large. The difference between the PHENIX [18, 19] and STAR $[12,20]$ non-photonic electron measurements appears to have been resolved [21]. However, the difference in the reported total $c \bar{c}$ cross sections seems to remain.

The calculated total charm cross section is strongly dependent on the charm quark mass and the renormalization and factorization scales [22]. The scale dependence is stronger than the mass dependence. We take $m_{c}=1.5 \mathrm{GeV}$ and $m_{b}=4.75 \mathrm{GeV}$ as the central values with $1.3 \leq m_{c} \leq 1.7 \mathrm{GeV}$ and $4.5 \leq m_{b} \leq 5 \mathrm{GeV}$ to estimate the mass uncertainties. The perturbative calculation also depends on the unphysical factorization $\left(\mu_{F}\right)$ and renormalization $\left(\mu_{R}\right)$ scales. (These scale parameters are unphysical in the sense that the real cross section should be independent of the scale.) The sensitivity of the cross section to their variation can be used to estimate the perturbative uncertainty due to the absence of higher orders. Since the partonic total cross section is independent of the kinematics, we take $\mu_{R, F}=\mu_{0}=m$ as the central value and varied the two scales independently within a 'fiducial' region defined by $\mu_{R, F}=$ $\xi_{R, F} \mu_{0}$ with $0.5 \leq \xi_{R, F} \leq 2$ and $0.5 \leq \xi_{R} / \xi_{F} \leq 2$. In practice, we use the following seven sets: $\left\{\left(\xi_{R}, \xi_{F}\right)\right\}$ $=\{(1,1),(2,2),(0.5,0.5),(1,0.5),(2,1),(0.5,1),(1,2)\}$. The uncertainties from the mass and scale variations are added in quadrature. The envelope containing the resulting curves,

$$
\begin{aligned}
\sigma_{\max } & =\sigma_{\mathrm{c}} \\
& +\sqrt{\left(\sigma_{\mu, \max }-\sigma_{\mathrm{c}}\right)^{2}+\left(\sigma_{m, \max }-\sigma_{\mathrm{c}}\right)^{2}} \\
\sigma_{\min } & =\sigma_{\mathrm{c}} \\
& -\sqrt{\left(\sigma_{\mu, \text { min }}-\sigma_{\mathrm{c}}\right)^{2}+\left(\sigma_{m, \min }-\sigma_{\mathrm{c}}\right)^{2}}
\end{aligned}
$$

defines the uncertainty as a function of energy. Here $\sigma_{\mathrm{c}}$ is the cross section calculated at the central value, $\left(\xi_{R}, \xi_{F}\right)=(1,1)$ and $m_{c}=1.5 \mathrm{GeV}, m_{b}=4.75 \mathrm{GeV}$ while $\sigma_{i, \max }$ and $\sigma_{i, \min }$ are the maximum and minimum values of the cross section for a given mass $(i=m)$ or $\left(\xi_{R}, \xi_{F}\right)$ set in the fiducial region $(i=\mu)$. Although Eqs. (1) and (2) have been written for the total cross section, the corresponding maximum and minimum differential distributions can be written similarly [23]. When the uncertainty on the $p_{T}$ distribution is calculated in the FONLL approach [24], $\mu_{0}$ is $m_{T}=d s q r m^{2}+p_{T}^{2}$ instead of $m$.
The results on the total cross section for charm and bottom are shown as a function of center of mass energy in Fig. 1. The solid curve shows the central result while the dashed lines show the upper and lower limits on the cross section, as calculated from the total partonic cross sections. The upper limit of the charm cross section at high energy is dominated by the contribution from $\left\{\left(\xi_{R}, \xi_{F}\right)\right\}=(0.5,1)$ due to the large value of $\alpha_{s}$ with 3 active quark flavors. On the other hand, the lower limit, with the flattening for $\sqrt{s}=200 \mathrm{GeV}$, is dominated by $\left\{\left(\xi_{R}, \xi_{F}\right)\right\}=(1,0.5)$ since CTEQ6M PDFs [25, 26] have been evolved below the minimum scale of the PDFs.

The FONLL integrated charm cross sections are somewhat lower because the FONLL approach, both for the fixed-order part and the resummed part, assumes that the charm quark is an active flavor, thus reducing the value of $\alpha_{s}$. In addition, the FONLL total cross section is based on the integrated $p_{T}$ and rapidity distributions, thus the average value of the factorization scale is at the average value of the $p_{T}$ distribution and thus higher than the factorization scale for the total partonic cross section. We note, however, that if the number of active flavors in the FONLL calculation is set to 3 for charm, the results of the two calculations are in very good agreement.

There is less disagreement for the bottom cross sections since the mass is higher. Thus the range of $\alpha_{s}$ explored by the parameter space is smaller while the factorization scale is well above the minimum scale of CTEQ6M $[25,26]$. The values of the cross sections at $\sqrt{s}=200 \mathrm{GeV}$ and $5.5 \mathrm{TeV}$ are shown in Table 1 . The CTEQ6M results are compared to results with the older GRV98 PDFs [27]. While this set is outdated, it shows how the width of the uncertainty band can be reduced by choosing a PDF set with a lower initial scale which reduces the factorization scale dependence and a smaller value of $\Lambda_{\mathrm{OCD}}^{n_{f}=5}$ which reduces the value of $\alpha_{s}$. For more details, see Refs. [22, 28].

The FONLL approach [24] is an excellent tool for calculating inclusive heavy flavor observables. It calculates not only heavy quark observables but also the heavy meson and semileptonic decay lepton observables. For example, if the inclusive electron spectrum from heavy flavor decay is measured, the calculation of the cross section involves three components: the $p_{T}$ and rapidity distributions of the heavy quark $Q$, calculated in perturbative QCD; fragmentation of the heavy quarks into heavy hadrons, $H_{Q}$, described by phenomenological input extracted from $e^{+} e^{-}$data; and the decay of $H_{Q}$ into electrons according to spectra available from other 

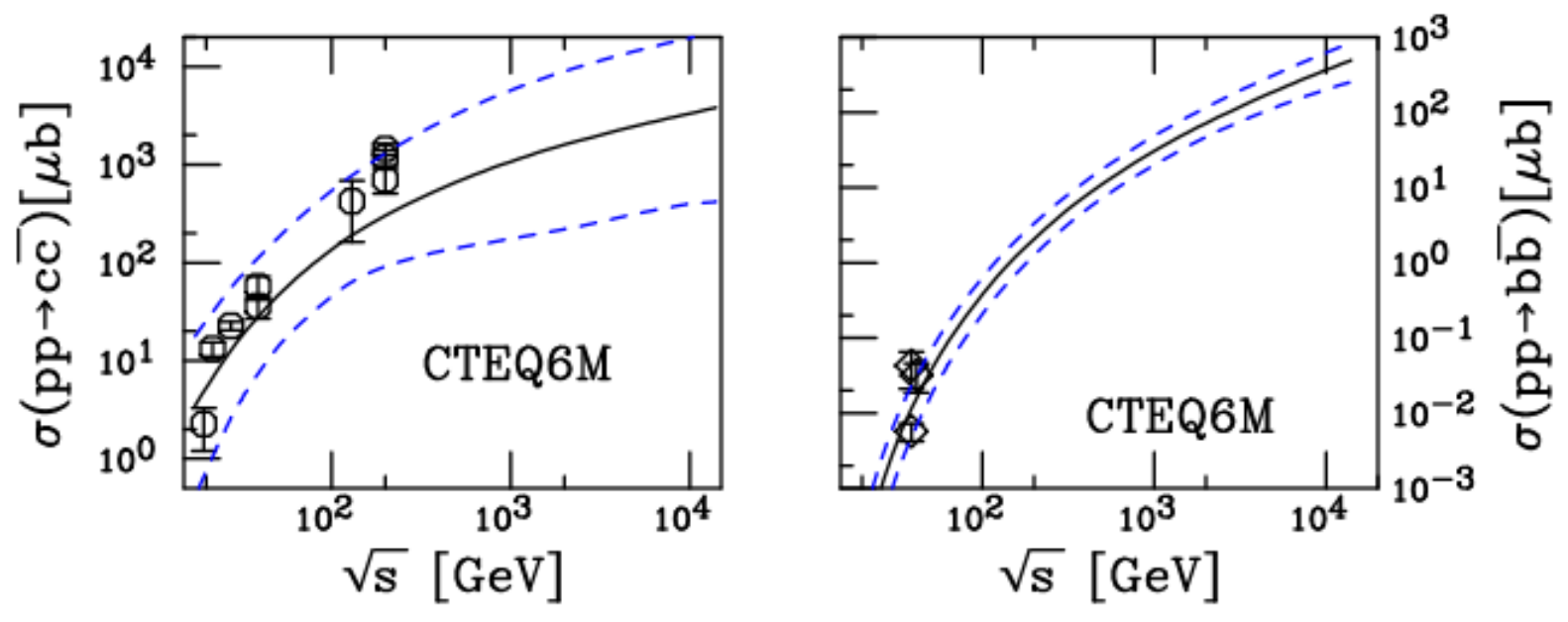

Figure 1: The NLO total $Q \bar{Q}$ cross sections as a function of $\sqrt{s}$ with CTEQ6M for charm (left) and bottom (right). The solid curve is the central result; the upper and lower dashed curves are the upper and lower edges of the uncertainty band.

\begin{tabular}{|l|lll||ll|}
\hline & \multicolumn{3}{|l||}{$\sqrt{S}=200 \mathrm{GeV}$} & $\sqrt{S}=5.5 \mathrm{TeV}$ \\
\hline$Q$ & $\sigma_{\text {CTEQ6M }}^{\text {FONLL }}(\mu \mathrm{b})$ & $\sigma_{\text {CTEQ6M }}^{\mathrm{NLO}}(\mu \mathrm{b})$ & $\sigma_{\text {GRV98 }}^{\mathrm{NLO}}(\mu \mathrm{b})$ & $\sigma_{\text {CTEQ6M }}^{\mathrm{NLO}}(\mu \mathrm{b})$ & $\sigma_{\text {GRV98 }}^{\mathrm{NLO}}(\mu \mathrm{b})$ \\
\hline$c$ & $256_{-146}^{+400}$ & $301_{-210}^{+1000}$ & $178_{-122}^{+300}$ & $2585_{-2260}^{+13125}$ & $3562_{-3321}^{+7321}$ \\
$b$ & $1.87_{-0.67}^{+0.99}$ & $2.06_{-0.81}^{+1.25}$ & $1.65_{-0.53}^{+0.77}$ & $209_{-84}^{+139}$ & $178_{-64}^{+93}$ \\
\hline
\end{tabular}

Table 1: Summary of the uncertainty on the charm and bottom total cross sections calculated from the NLO partonic total cross sections at RHIC and the LHC.

measurements, schematically written as

$\frac{E d^{3} \sigma(e)}{d p^{3}}=\frac{E_{Q} d^{3} \sigma(Q)}{d p_{Q}^{3}} \otimes D\left(Q \rightarrow H_{Q}\right) \otimes f\left(H_{Q} \rightarrow e\right)$

where the symbol $\otimes$ denotes a generic convolution. The electron decay spectrum, $f\left(H_{Q} \rightarrow e\right)$, accounts for the semileptonic branching ratios. Note, however, that the FONLL approach cannot calculate exclusive $Q \bar{Q}$ pair observables.

While the differences between the STAR and PHENIX non-photonic electron spectra have been reduced, full resolution requires separate measurements of open charm and bottom hadrons. There are several analysis methods available for this separation but direct measurement of charm and bottom hadrons is, of course, preferable. The prospects for such measurements at RHIC II are discussed in Ref. [1].

Analyses that separate leptons from charm and bottom decays are currently available, based mainly on lepton-hadron correlations. PHENIX also has an early result on $e \mu$ correlations [29], suggested as a method of separating correlated heavy flavor production [30].

The azimuthal correlations between non-photonic electrons and charged hadrons $[31,32]$ as well as between non-photonic electrons and $D^{0}$ mesons [32] have been used to obtain the fraction of the non-photonic electrons coming from semileptonic $B$ decays. Simulations show that the width of the near-side $(\Delta \phi \sim 0) e-h$ correlation is larger for $B$ decays than $D$ decays because more energy is released in $B$ decays [32]. The fraction of non-photonic electrons expected from $B$ decays relative to $D$ decays is consistent with expectations from FONLL [31, 32].

The $e-D^{0}$ azimuthal correlations provide an independent measurement. Decays of $c \bar{c}$ pairs make predominantly like-sign, away-side $(\Delta \phi \sim \pi)(\bar{D} \rightarrow) e^{-}$$D^{0}\left(\rightarrow K^{-}\right)$and $e^{+}-\bar{D}^{0}\left(\rightarrow K^{+}\right)$pairs. On the other hand, $b \bar{b}$ decays make like-sign, near-side $e-K$ pairs through a single $B^{-} \rightarrow e^{-} \bar{v}_{e} D^{0}\left(\rightarrow K^{-}\right)$decay chain. Oppositesign, away-side $e-K$ pairs are produced from $\left(B^{-} \rightarrow\right) e^{-}$- 
$B^{+} \rightarrow \bar{D}^{0}\left(\rightarrow K^{+}\right)$decays. Thus like-sign $e-K$ pairs select $b \bar{b}$ decays on the near side and $c \bar{c}$ decays on the away side [33]. The relevant decay chains are schematically illustrated in Fig. b.
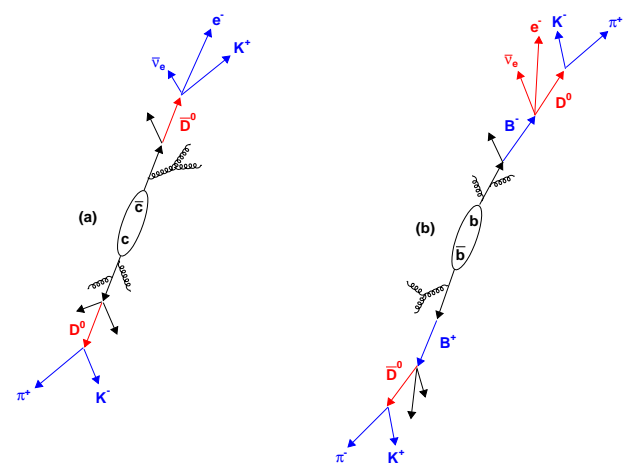

Figure 2: Schematic decay chains for $c \bar{c}$ (a) and $b \bar{b}$ (b) to like- and opposite-sign $e-K$ pairs.

More directly, $B$ mesons decay to $J / \psi$, contributing to high $p_{T} J / \psi$ production. The first STAR result on the fraction of $J / \psi$ production from $B$ decays suggests $13 \pm 5 \%$ of $J / \psi$ 's with $p_{T}>5 \mathrm{GeV}$ come from $B$ mesons [34]. This result, along with new, higher statistics STAR data [35], is consistent with the CDF measurement at $1.96 \mathrm{TeV}$ [36]. It is important to separate the secondary $J / \psi$ coming from $B$ decays from the prompt $J / \psi$ production because the in-medium effects will be different for the two $J / \psi$ sources.

Thus while the theoretical uncertainties on open heavy flavor production can be large, a good understanding of heavy flavor production provides an important handle on the total quarkonium production rates. In $A A$ collisions, the open heavy flavor rate sets the normalization for quarkonium recombination [14]. Separation of leptons from charm and bottom semileptonic decays will also help to understand the importance of the quark mass on the relative energy loss [3].

\section{Acknowledgments}

The work of R.V. was performed under the auspices of the U.S. Department of Energy by Lawrence Livermore National Laboratory under Contract DE-AC5207NA27344 and was also supported in part by the National Science Foundation Grant NSF PHY-0555660.

\section{References}

[1] A. D. Frawley, T. Ullrich and R. Vogt, Phys. Rept. 462 (2008) 125 [arXiv:0806.1013 [nucl-ex]].

[2] J. F. Amundson, O. J. P. Eboli, E. M. Gregores and F. Halzen, Phys. Lett. B 390 (1997) 323 [arXiv:hep-ph/9605295].

[3] M. Djordjevic, M. Gyulassy, R. Vogt and S. Wicks, Phys. Lett. B 632 (2006) 81 [arXiv:nucl-th/0507019].

[4] M. Djordjevic, M. Gyulassy, and S. Wicks, Phys. Rev. Lett. 94 (2005) 112301 [arXiv:hep-ph/0410372].

[5] N. Armesto, C. A. Salgado, and U. A. Wiedemann, Phys. Rev. D 69 (2004) 114003 [arXiv:hep-ph/0312106].

[6] Yu. L. Dokshitzer and D. E. Kharzeev, Phys. Lett. B 519 (2001) 199 [arXiv:hep-ph/0106202].

[7] Z.-W. Lin, R. Vogt and X.-N. Wang, Phys. Rev. C 57 (1998) 899 [arXiv:nucl-th/9705006]; Z.-W. Lin and R. Vogt, Nucl. Phys. B 544 (1999) 339 [arXiv:hep-ph/9808214].

[8] R. Vogt [Hard Probe Collaboration], Int. J. Mod. Phys. E 12 (2003) 211 [arXiv:hep-ph/0111271].

[9] B. Svetitsky and A. Uziel, Phys. Rev. D 55 (1997) 2616 [arXiv:hep-ph/9606284].

[10] V. Greco, C. M. Ko and R. Rapp, Phys. Lett. B 595 (2004) 202 [arXiv:nucl-th/0312100].

[11] Z.-W. Lin and D. Molnar, Phys. Rev. C 68 (2003) 044901 [arXiv:nucl-th/0304045].

[12] B. I. Abelev et al. [STAR Collaboration], Phys. Rev. Lett. 98 (2007) 192301 [arXiv:nucl-ex/0607012].

[13] A. Adare et al. [PHENIX Collaboration], Phys. Rev. Lett. 98 (2007) 172301 [arXiv:nucl-ex/0611018].

[14] R. L. Thews and M. L. Mangano, Phys. Rev. C 73, 014904 (2006) [arXiv:nucl-th/0505055].

[15] R. L. Thews, M. Schroedter, and J. Rafelski, Phys. Rev. C 63 (2001) 054905 [arXiv:hep-ph/0007323].

[16] A. Andronic, P. Braun-Munzinger, K. Redlich and J. Stachel, Phys. Lett. B 571 (2003) 36 [arXiv:nucl-th/0303036].

[17] A. P. Kostyuk, M. I. Gorenstein, H. Stöcker and W. Greiner, Phys. Rev. C 68 (2003) 041902 [arXiv:hep-ph/0305277].

[18] A. Adare et al. [PHENIX Collaboration], Phys. Rev. Lett. 97 (2006) 252002 [arXiv:hep-ex/0609010].

[19] S. S. Adler et al. [PHENIX Collaboration], Phys. Rev. Lett. 96 (2006) 032301 [arXiv:nucl-ex/0510047].

[20] J. Adams et al. [STAR Collaboration], Phys. Rev. Lett. 94 (2005) 062301 [arXiv:nucl-ex/0407006].

[21] W. Xie [STAR Collaboration], in proceedings of Hard Probes 2010, Eilat, Israel, October 2010.

[22] R. Vogt, Eur. Phys. J. ST 155 (2008) 213 [arXiv:0709.2531 [hep-ph]].

[23] M. Cacciari, P. Nason and R. Vogt, Phys. Rev. Lett. 95 (2005) 122001 [arXiv:hep-ph/0502203].

[24] M. Cacciari, M. Greco and P. Nason, JHEP 9805 (1998) 007 [arXiv:hep-ph/9803400]; M. Cacciari, S. Frixione and P. Nason, JHEP 0103 (2001) 006 [arXiv:hep-ph/0102134].

[25] J. Pumplin, D. R. Stump, J. Huston, H. L. Lai, P. M. Nadolsky and W. K. Tung, JHEP 0207 (2002) 012 [arXiv:hep$\mathrm{ph} / 0201195]$

[26] D. Stump, J. Huston, J. Pumplin, W. K. Tung, H. L. Lai, S. Kuhlmann and J. F. Owens, JHEP 0310 (2003) 046 [arXiv:hep-ph/0303013].

[27] M. Glück, E. Reya and A. Vogt, Eur. Phys. J. C 5, 461 (1998) [arXiv:hep-ph/9806404].

[28] R. Vogt, Eur. Phys. J. C 61 (2009) 793.

[29] T. Engelmore [PHENIX Collaboration], Nucl. Phys. A 830 (2009) 853C [arXiv:0907.4813 [nucl-ex]].

[30] S. Gavin, P. L. McGaughey, P. V. Ruuskanen and R. Vogt, Phys. Rev. C 54, 2606 (1996) [arXiv:hep-ph/9604369]. 
[31] A. Adare et al. [PHENIX Collaboration], Phys. Rev. Lett. 103 (2009) 082002 [arXiv:0903.4851 [hep-ex]].

[32] M. M. Aggarwal et al. [STAR Collaboration], arXiv:1007.1200 [nucl-ex].

[33] A. Mischke, Phys. Lett. B 671 (2009) 361 [arXiv:0807.1309 [hep-ph]].

[34] B. I. Abelev et al. [STAR Collaboration], Phys. Rev. C 80 (2009) 041902 [arXiv:0904.0439 [nucl-ex]].

[35] Z. Tang [STAR Collaboration], in proceedings of Hard Probes 2010, Eilat, Israel, October 2010.

[36] D. Acosta et al. [CDF Collaboration], Phys. Rev. D 71 (2005) 032001 [arXiv:hep-ex/0412071]. 